\title{
The Kinetic Stabilizer: Further Calculations and Options
}

Richard F. Post

This article was submitted to $4^{\text {th }}$ International Conference on Open Magnetic Systems for Plasma Confinement, Jehu Island, Korea, July 1-4, 2002

\section{June 19, 2002}

U.S. Department of Energy

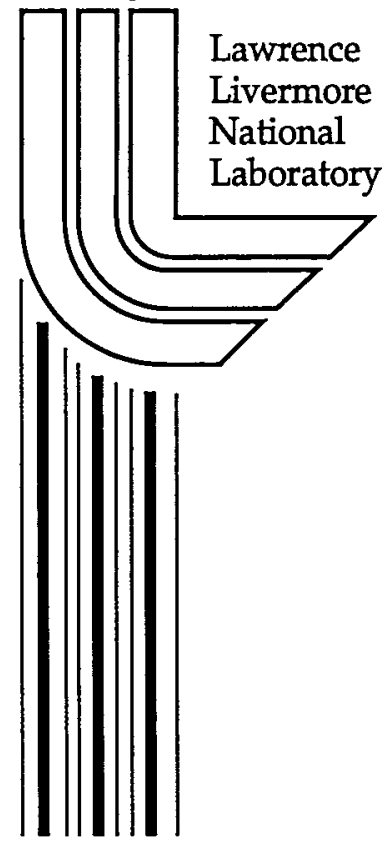




\section{DISCLAIMER}

This document was prepared as an account of work sponsored by an agency of the United States Government. Neither the United States Government nor the University of California nor any of their employees, makes any warranty, express or implied, or assumes any legal liability or responsibility for the accuracy, completeness, or usefulness of any information, apparatus, product, or process disclosed, or represents that its use would not infringe privately owned rights. Reference herein to any specific commercial product, process, or service by trade name, trademark, manufacturer, or otherwise, does not necessarily constitute or imply its endorsement, recommendation, or favoring by the United States Government or the University of California. The views and opinions of authors expressed herein do not necessarily state or reflect those of the United States Government or the University of California, and shall not be used for advertising or product endorsement purposes.

This is a preprint of a paper intended for publication in a journal or proceedings. Since changes may be made before publication, this preprint is made available with the understanding that it will not be cited or reproduced without the permission of the author.

This report has been reproduced directly from the best available copy.

Available electronically at http://www.doc.gov/bridge

Available for a processing fee to U.S. Department of Energy

And its contractors in paper from

U.S. Department of Energy

Office of Scientific and Technical Information

P.O. Box 62

Oak Ridge, TN 37831-0062

Telephone: (865) 576-8401

Facsimile: (865) 576-5728

E-mail: reports@adonis.osti.gov

Available for the sale to the public from

U.S. Department of Commerce

National Technical Information Service

5285 Port Royal Road

Springfield, VA 22161

Telephone: (800) 553-6847

Facsimile: (703) 605-6900

E-mail: orders@ntis.fedworld.gov

Online ordering: http://www.ntis.gov/ordering.htm

\section{OR}

Lawrence Livermore National Laboratory

Technical Information Department's Digital Library

http://www.llnl.gov/tid/Library.html 


\title{
THE KINETIC STABILIZER: FURTHER CALCULATIONS AND OPTIONS
}

\author{
Richard F. Post \\ Lawrence Livermore National Laboratory \\ 7000 East Avenue, L-644, Livermore, CA 94551 \\ (925) 422-9853, post3@1lnl.gov
}

\begin{abstract}
The Kinetic Stabilizer, employing injected and mirror-reflected ion beams, represents a method for stabilizing axisymmetric mirror and tandem mirror systems. Building on earlier work, this paper presents further calculations on the concept and explores some new options that promise to enhance its capabilities
\end{abstract}

\section{1) INTRODUCTION}

The magnetic confinement approach to fusion power has been under study for more than fifty years. During these many years enormous progress has been made in the understanding of the physics of plasmas and their complex interactions with magnetic fields. Despite this progress and despite the growing awareness of the importance of fusion as a source of energy, the goal of achieving an energetically self-sustaining fusion reaction in a magnetically confined plasma still eludes us. In assessing the barriers that have prevented this goal from being reached a single factor stands out: That is, the dominant role that plasma turbulence has played in constraining the course of fusion research, including its effect on the programmatic decisions that have been made by the fusion research community and by the governmental agencies supporting them.

Today magnetic fusion research is presented with both an opportunity and a dilemma. The dilemma: if the research continues on its present course, based on the front-runner tokamak, it is clear that it will be many years before even one demonstration of net fusion power can occur. Furthermore, it is not clear that this demonstration will lead automatically to practical fusion power systems. The opportunity: there should be contained, in the body of knowledge about plasma physics accumulated in the last fifty years, the seeds of a simpler, smaller, soonerachieved avenue to the fusion goal. This paper addresses issues relating to one possible candidate for this kind of a new look at the magnetic fusion approach.

Because the story may be unfamiliar to the fusion research community of today, and because it is relevant to the issues to be discussed, a brief historical review, telling how we got to the present situation in magnetic fusion research, will be given. Following that discussion, some seminal theoretical and experimental developments in the 1990s, developments that led to the study of the Kinetic Stabilizer concept [1], will be discussed. Finally, some new calculations and suggested options for implementing the Kinetic Stabilizer idea as applied to a tandem-mirror system will be presented.

\section{2) FUSION HISTORY: THE PAST IS PROLOGUE}

From the earliest days there were understood to be two topologically distinct approaches to magnetic fusion, the closed and open approaches, referring to the geometry of the magnetic field lines. These two different approaches represent different responses to what was early on understood as the problem of the ends, that is, the fact that a uniform magnetic field provides no constraint against axial motion of charged particles that are trapped on its field lines. The most obvious solution to this problem is to close the field lines on themselves, for example to constrain them to lie within a topological torus, thus becoming a closed system. The less obvious solution, using concepts borrowed from astrophysics [2], was the open magnetic mirror approach, where the free flow of particles out the ends of a tube of magnetic flux is plugged by increasing the strength of the field at the ends (the mirrors ). As history reveals, both the closed and the open approaches have had instability-related limitations and flaws that have slowed their progress toward solving the fusion problem.

First studied, and first to fall victim to plasma instability, was the pinch-effect, an early closed-field system based on confinement by the magnetic field produced solely by currents flowing in the plasma itself. The next approach to have its confinement spoiled by plasma turbulence was Spitzer s Stellarator, a closed system using externally generated magnetic fields, formed, in its first embodiment, into a figure- 8 configuration. . Known as pump-out at the time, the plasma exhibited diffusion rates across its confining field at a turbulenceenhanced rate approaching that predicted by David Bohm, 
five or more orders of magnitude faster than the rate predicted by Spitzer [3] for collision-induced fusion across a uniform magnetic field.

At the same time that these disappointing results were being obtained in closed systems, instability-related problems were being encountered in early mirror machines. One of the earliest encountered was the interchange MHD instability, endemic to the first mirror machines, the magnetic fields of which were axisymmetric, (i.e., fields generated by coaxial circular loop currents). As shown early on theoretically by Rosenbluth and Longmire [4], the interchange instability arises from a competition between the destabilizing effect of the negative-curvature region of the field lines between the mirrors and the (weaker) stabilizing effect of the positive-curvature regions near the mirrors. The solution (at the time) to this problem was shown by the elegant experiment performed in 1961 by Ioffe [5] in which he modified the shape of the field, by employing currents flowing in longitudinal Ioffe bars. The effect of the Ioffe bars was to make the curvature of the field lines bounding the plasma everywhere positive, i.e., the plasma was contained in a magnetic well. Following Ioffe $s$ experiment, virtually every mirror machine in the world fusion community was converted to use some form of magnetic-well field, either generated by Ioffe bars or by baseball or yin-yang [5] coils. As will later be discussed the abandonment of axisymmetry was a mixed blessing.

Having suppressed MHD instabilities, the mirror community next had to deal with another class of instabilities, the so-called loss-cone instabilities, originating from the anisotropic and non-maxwellian nature of plasmas confined by the mirror effect. In time these instabilities were understood theoretically, including prediction of the means for stabilizing them [6]. When these means were implemented experimentally in magnetic-well-type mirror cells both the MHD and the loss-cone instabilities were suppressed, leading to spectacular improvement in the confinement, a direct result of the absence of turbulence-related transport. The plasma was stable, even at plasma pressures approaching that of the confining field, i.e. beta approaching unity. These results, obtained in the 1970s in the 2 XIIB experiment [7] at Livermore, were extremely encouraging, but they still left open the issue of collision-induced leakage through the mirrors, which even in a quiescent plasma would make the fusion power balance marginal. Even with the addition of means of direct conversion of the end-escaping particle fluxes [8] to increase the effective $Q$ of the confinement, fusion power systems relying only on mirror confinement would be severely limited by the effects of end losses.

For closed systems, in the 1970s a history-defining event occurred when Lev Artsimovich of the Soviet Union made a pilgrimage to spread the word concerning results obtained in Russia with the tokamak [9]. As every school student today should know, the tokamak uses a combination of magnetic fields generated by coils wound on the surface of a torus and those arising from transformer-induced currents flowing along the helical field lines within the torus. Though still exhibiting turbulence-enhanced cross-field transport, the tokamak demonstrated confinement that was two orders of magnitude better than the Bohm-dominated diffusion in the stellarators of that day. More significant for advocates, the empirical transport scaling laws that were developed in succeeding generations of tokamaks projected to fusionrelevant confinement times for a sufficiently large device. Thus there began a shift toward tokamak research that soon would became a landslide, one as a result of which effort devoted to alternative approaches either would disappear completely or drop to a very low level, world-wide.

During this transition period, in 1976, the mirror approach got a new lease on life as a result of the invention of the tandem-mirror concept, originated independently by Dimov in the Soviet Union [10] and Fowler and Logan in the United States [11]. The tandem-mirror idea, if it could be implemented practically, offered an effective means of controlling end losses in mirror machines. First experiments, at Novosibirsk, Livermore, and Tsukuba in Japan, were very encouraging, leading to the design of new and/or upgraded tandem-mirror facilities in all three countries. By the mid-1980s a very large tandem-mirror system, MFTF (for Mirror Fusion Test Facility) had been built at the Lawrence Livermore National Laboratory with high hopes for its performance. However, simultaneously with the completion and shakedown tests of MFTF, the U. S. fusion program suffered a severe budget cut. The response of the governmental bureau that supported magnetic fusion research was to eliminate its support of all approaches except the tokamak. Worldwide only Novosibirsk and Tsukuba, (and, more recently, Korea) have carried on with tandem-mirror research.

Except for a few pockets of resistance, during the last decade of the twentieth century the world s magnetic fusi;on effort was dominated by the tokamak. Relying on empirically derived scaling laws, ever-larger tokamak facilities were constructed in a drive toward the achievement of plasma temperatures, densities, and confinement times that would approach the goal of plasma ignition Achievements, as measured by the generation of multi-megawatts of fusion power release in TFTR at Princeton and JET at Culham, led to the proposal of a really huge tokamak, ITER (for International Thermonuclear Experimental Reactor). Because its projected cost was so large ITER could only be undertaken as a decades-long multi-national project. As of today final decisions concerning siting have not been made, and a 
major original partner that withdrew several years ago (the United States) appears ambivalent about rejoining the ITER team. Perhaps some of this apparent ambivalence arises from the concern that the size, cost, and complexity of ITER is such as to raise questions about whether the tokamak approach will in fact lead to a practical and economically viable fusion power system. Also possibly as a result of this concern, in recent years the US magnetic fusion effort has been directed by Congress to place a greater emphasis on plasma physics issues, particularly those that might lead to simpler, smaller, and sooner achieved approaches to fusion power.

\section{3) ORIGINS OF THE KINETIC STABILIZER IDEA: AXISYMMETRY AND THE GAS DYNAMIC TRAP}

Among the pockets of resistance to the tokamak "feeding frenzy" is the Budker Institute in Novosibirsk. Budker having been an inventor of the mirror machine concept, for many years this group has been studying mirror-based systems, particularly including ones with axisymmetric fields. In the late 1980s Institute plasma theorist Dmitri Ryutov analyzed a novel means for stabilizing axisymmetric mirror systems against the MHD interchange instability [12]. His analysis was beautifully confirmed in the Gas Dynamic Trap experiment at his laboratory, at plasma beta values of 30 percent [13] (almost an order of magnitude higher than typical tokamak values). The concept he analyzed: plasma escaping out the ends of an axisymmetric mirror system can stabilize plasma in the interior if it is allowed to expand sufficiently far in the diverging field lines outside the mirror, even when the density of the effluent plasma is much less than that of the interior, confined plasma. Not only was the plasma stabilized, but measurements confirmed that the rate of diffusion of the confined plasma across the magnetic field was far below the turbulence-enhanced rates of closed systems, approaching the classical, Spitzer, value.

The good confinement results obtained in the axisymmetric GDT experiment gave solid proof of results hinted at in very early mirror experiments, such as the 1960s Table Top mirror experiment at Livermore [14]. In this axisymmtric mirror experiment a metastable spindleshaped, $20 \mathrm{keV}$, hot-electron plasma was created by magnetic compression. This plasma was observed to have a radial diffusion rate that was five orders of magnitude slower than the Bohm diffusion rate.

Ryutov s theory and the GDT thus opened the door to the use of axisymmtric fields in open systems, but it came with a caveat: although the density of the effluent, stabilizing, plasma can be much lower than that of the confined plasma, it must be high enough. In the GDT, which operates in a high-density, high collisionality, regime, the effluent plasma, though much lower in density than the interior plasma, is still high enough to satisfy the stability criterion. But in a low-collisionality situation such as would be encountered in a tandem-mirror system, the effluent plasma density would be too low to satisfy the stability criterion. The Kinetic Stabilizer [1] represents s possible way to resolve this dilemma, and thereby to permit the design of tandem-mirror systems with only axisymmetric fields. If this became possible there would be many predicted advantages for an axisymmetric tandem-mirror system (as compared to ones that rely on non-axisymmetric fields for MHD stability), both in improved confinement, and in economic and engineeringrelated issues.

\section{4) THE KINETIC STABULZER CONCEPT}

The Kinetic Stabilizer concept as applied to axisymmetric mirror-based systems has been described in a previous paper [1]. Its starting point was an earlier concept, the Kinetic Tandem [15]. The idea of the Kinetic Stabilizer is to create in situ a localized plasma on the expanding field lines (the expander) lying outside the outermost mirror of an axisymmetric tandem-mirror system. This localized plasma is to be created by the kinetic technique of launching directed ion beams from ion sources lying still farther out on the expanding field lines. These ions, aimed at small angles to the local direction of the field lines, would be compressed, stagnated, and reflected at a pre-determined position on the converging field lines, chosen so as to optimize the stabilizing effect of the beam-produced plasma. What was shown in the previous paper is that, when optimally produced, the density of this stabilizer plasma could be many orders of magnitude lower than that of the plug plasma in a tandem-mirror system, and still be effective in MHD-stabilizing that plasma.

The stagnation and reflection of ions injected up the magnetic gradient in the expander is governed by a simple relationship, derived from adiabatic invariance of the magnetic moment and the particle energy (ignoring potential effects, which can be later introduced selfconsistently). The relationship between the angle of injection relative to the direction of the the field lines at the launching point and the ratio of the field at the reflection point to the field at the launching point is the simple mirror formula, Equation 1.

$$
\frac{B_{\text {refl }}}{B_{i n j}}=\frac{1}{\sin ^{2}\left(\theta_{i n j}\right)}
$$

The same two invariants can be used to calculate the shape and compression of the density peak formed by the injection of an ion beam having a prescribed angle distribution $[1,15]$. 
The field-line-curvature-related stability criterion that must be satisfied by the Kinetic Stabilizer plasma can be seen from an examination of the MHD stabilization criterion for an axisymmetric mirror system [12], stated in integral form in Equation 2.

$$
I_{s}=\int_{-L}^{L} a^{3} \frac{d^{2} a}{d z^{2}}\left[p_{\text {perp }}+p_{p a r}+\rho v^{2}\right] d z>0 \text {, Stable }
$$

In this expression the radius of the plasma is represented by the term, a. The integral is to be carried out over the length of the plasma between the end walls of the system, located at $-\mathrm{L}$ and $+\mathrm{L}$, respectively. The term in the brackets represents the total kinetic pressure of the plasma (a function of position). This pressure term is then multiplied by the plasma radius cubed and the second derivative of the plasma radius (the curvature term) and then integrated over the length of the system to determine the sign of $I_{s}$.

As can be seen from Equation 2, regions of the plasma at large radius and where the field-line curvature is also strongly positive will make the largest positive (stabilizing) contributions to the integral. The Kinetic Stabilizer takes advantage of this scaling by creating its kinetically produced plasma at an optimally chosen location on the region of expanding field lines (the expander) outside the mirrors, achieved by aiming the ion beams so that they will be mirror-reflected within this region. The optimizations required are accomplished by designing the field coils in the expander so as to create flux surfaces that concentrate the regions of positive curvature at a position intermediate between the mirror field and the end region of the expander where the ion sources are located. In this way it is possible to take the best advantage both of the scaling with radius and curvature and of the magnetic compression that results as the injected ions move into an increasing field.

\section{5) EVALUATION OF THE STABLITY INTEGRAL}

As described in a previous paper [1] a computer code was written that can be used to evaluate the instability integral, Equation 2, both in the confined plasma and in the expander, including the formation of the density peak from the injected ion beams. As an example, in Reference 1 a case was calculated for $\mathrm{s}$ simple mirror cell (fusion $\mathrm{Q}<1$ ), confining a fusion plasma producing $45 \mathrm{MW}$ of fusion energy (as might be the case for a fast-neutron source used for testing radiation-damage effects). It was found that this plasma could be stabilized by the injection of 200 kilowatts of $1 \mathrm{keV} \mathrm{Cs}{ }^{+}$ions. This result illustrates the strong stabilizing effect that can be exerted by a properly positioned stabilizer plasma peak generated in the expander.
For an axisymmetric tandem-system the situation would be quantitatively different, and the stabilizer beampower requirements, dictated primarily by the destabilizing contributions from the plug cells, require that one minimize the negative contributions by shaping the flux surfaces in the plug cells in an optimized manner, and by minimizing the negative contribution of the plug plasma to the stability integral by controlling its distribution functions. A double gain can be obtained if a "sloshingion" distribution function [16] is employed. Such a distribution, which has been employed successfully previously in mirror experiments [17] has the dual effect of suppressing the Alfv n Ion Cyclotron instability, while at the same time reducing the negative contribution to the stability integral. The former occurs because the sloshingion distribution is less anisotropic (less unstable for the AIC mode) than a "normal-mode" distribution. The latter occurs because the sloshing ion distribution has more of its ions exposed to the positive field line curvature regions of the cell.

As an example from earlier calculations [18], the flux surfaces of a tandem-mirror plug cell formed by a series of current loops, the currents in which increase linearly with distance from the midplane, were calculated and used as input to the stability integral. A typical flux surface for such a mirror cell is shown in Figure 1.

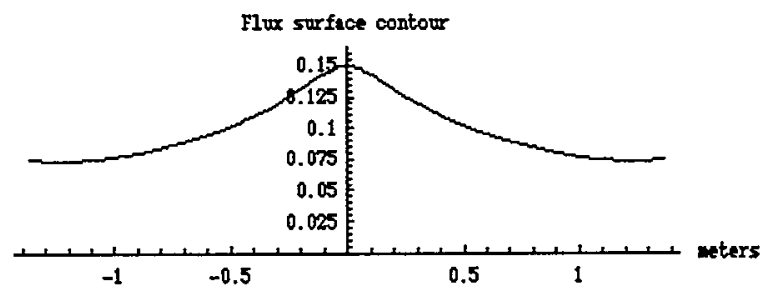

Figure 1: Flux surface associated with an assembly of circular current loops (radius $0.25 \mathrm{~m}$.) the currents in which increase linearly (from a base value) with distance from the midplane (note the change of scale between the $y$ and $x$ axes).

The shape of the flux surface shown in Figure 1 can be seen to be such that it is well-suited for the containment of a sloshing-ion type of distribution in that the sloshing ions will be preferentially reflected in regions of positive fieldline curvature. On the other hand, this flux surface would not be expected to be advantageous for the centrally peaked normal-mode distributions that would be characteristic of mirror-confined plasmas under usual circumstances. 
To illlustrate the gains (reductions in the negative contribution to the stability integral) that could be expected by employing sloshing-ion distributions in mirror cells with flux surfaces of the type shown in Figure 1 comparison calculations of the stability integral were made. First, a normal-mode distribution was used together with a conventional (Bessel-function type) of flux surface. The cell length was 2.5 meters, the mirror ratio was $4: 1$, and the radius of the flux surface at the midplane was $0.15 \mathrm{~m}$

The value of the instability integral was calculated for three cases: (1) for the normal-mode distribution (Figure 8 ) in the Bessel-function cell, (2) for a sloshing-ion distribution in the same cell, and (3) for a sloshing-ion distribution in the linear-taper cell of Figure 1. To illustrate the gains achievable by the optimization of the flux surfaces and by the use of sloshing ions, Table IV gives the value, $I_{s}$, of the stability integral for the three cases. The first entry is for the normal mode case in the Bessel-function cell.. The second entry is for the sloshingion distribution in the same cell. The third entry is for the sloshing-ion distribution in the linear-taper-coil cell of Figure 1. For all cases the mirror ratio was $4: 1$ and the plasma radius at the mirrors had the same value $(.075 \mathrm{~m}$.). All distributions were normalized to unity at their peak pressures so that all would generate the same peak plugging potential in a tandem-mirror system.

Table 1

Cell Type and Density Distribution

Normal-mode dist. in Bessel cell

Sloshing-ion dist. in Bessel cell

Sloshing-ion dist. in taper-coil cell
$\mathbf{I}_{\mathbf{s}}$

$-3.7 \times 10^{-4} \quad 1.03$

$-1.4 \times 10^{-4} \quad 0.39$ $-3.6 \times 10^{-4} \quad 1.0$

It can be seen from Table I that a substantial reduction in the negative contribution to the stability integral can be achieved by using sloshing ions in a taper-coil cell as compared to either normal-mode or sloshing-ion distributions contained in a Bessel-function cell. This reduction comes about as a result of the combined effect of sloshing-ions and the favorable shaping of the field lines that occurs when the cell employs the linear-taper configuration for the current in its field coils.

The example given above illustrates the kinds of reductions in destabilizing effect (reflected in reductions in the K-S beam-power requirements) that are possible by shaping the flux surfaces in the end-cells of a tandem mirror employing Kinetic Stabilizers. In a 1988 paper concerning the Gas Dynamic Trap, Mirnov and Ryutov [19] employed variational analysis to determine the optimal shape of the flux surfaces in the mirror cell of the GDT, that is, the shape that minimizes the negative contribution of this cell to the instability integral. Although in the case that they treated the plasma pressure was isotropic (owing to the high collisionality of the GDT operating regime), their analytical approach could also be applied to a sloshing-ion pressure distribution. In that way even further gains than those presented here could no doubt be realized, within the limits imposed by engineering requirements in the construction of the field coils.

\section{6) ISSUES CONNECTED WITH THE INJECTED ION BEAMS}

A key assumption implicit in calculating the position and density of the peaked stabilizer plasma is that the trajectories should be controlled by the invariants of the motion, i.e., magnetic moment and energy. To investigate this point for realistic cases an orbit code was written that traced out the orbits of ions injected into typical expander fields for various initial conditions at the point of injection. From these calculations it was determined that there is an optimum way to inject the ions to insure that their subsequent motion will come as close as possible to that predicted by the use of the invariants. If we consider a coordinate system in which the emergent field line on the expander flux surface defines a polar axis, then there was found to be an optimum azimuthal angle in this coordinate system This angle lies in the plane defined by the" $z$ " axis of the magnetic field and the emerging field line as it converges toward this axis. If we take the zero azimuthal angle in this system as lying in this plane for an ion whose velocity component perpendicular to the direction of the field line is aimed toward the $z$ axis, i.e. inward, this angle of injection results in the least deviation from the predictions of adiabatic theory. Table II compares the calculated mirror ratio at reflection with that predicted by adiabatic theory. The case that was calculated was for an expander that was 10.5 meters in length between the mirror and the location of the ion sources, and for which the magnetic field on axis decayed as a gaussian, with a characteristic length of 5.0 meters. The field at the mirror was 20 Tesla, falling off to $0.17 \mathrm{~T}$ at the position of the ion sources. The ions, which were singly ionized $0.25 \mathrm{keV}$ Krypton ions $(A=84)$, were injected at an angle to the local direction of the field lines of $17.5 \ldots$ at several different azimuthal angles. In the Table Column 1 lists the azimuthal angle of injection, $\phi$ (in the polar coordinate system defined by the local direction of the field line on which the ion is launched), Column 2 lists the observed mirror ratio at reflection, and Column 3 lists the ratio of the calculated orbit and the adiabatic reflection mirror ratios. For this case the reflection mirror ratio as calculated from the invariants is 11.06 , corresponding to a magnetic field strength at the plane of reflection of $1.94 \mathrm{~T}$ 
Table II

$\begin{array}{lcl}\phi & \mathbf{R}_{\text {calc. }} & \mathbf{R}_{\text {calc }} / \mathbf{R}_{\text {adiabatic }} \\ 0 \ldots & 11.29 & 1.021 \\ 30 \ldots & 15.99 & 1.446 \\ 60 \ldots & 21.24 & 1.921 \\ 90 \ldots & 21.81 & 1.972 \\ -30 \ldots & 8.51 & 0.770 \\ -60 \ldots & 7.10 & 0.642 \\ -90 \ldots & 6.59 & 0.596\end{array}$

As can be seen from the Table, injection at an azimuthal angle of $0 \ldots$ results in an orbit for which the reflection field agrees with the adiabatic result to within 2 percent, while large deviations from the adiabatic result occur for the other angles.

\section{7) EXPANDER DESIGN ISSUES}

As discussed in a previous paper [1] the design of the expander presents an opportunity to optimize the performance of the Kinetic Stabilizer. To this end a design was made where the fields were generated by a series of circular loop coils carrying currents that were tailored to generate the desired flux surface shape. The coils are wound on a double-conical surface the shape of which was chosen to approximately match the shape of the outermost flux surface. Figure 2 is a plot of the outline of these two surfaces, that is the loop coils and, inside them, the flux surface. Note that the flux surface is initially conical in shape (zero second derivative), followed by a region of large positive curvature.

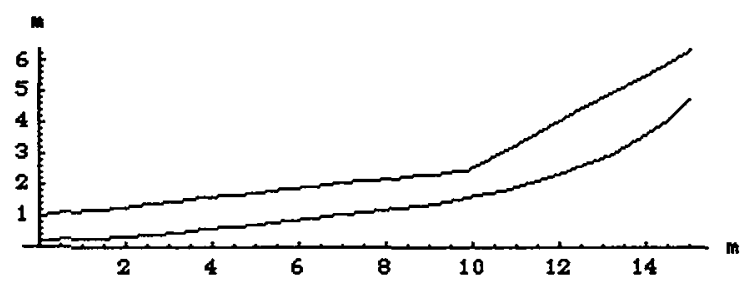

Figure 2: Outline of coils and the outer flux surface for the double-conical expander

The flux surface shown was employed in the stability integral code to evaluate the integral for ion source located at the end of the expander, aimed along the field lines with a quasi-gaussian angular distribution centered at an angle of $15 \ldots$ and with an angular spread of $5 \ldots$. The calculated density peaking produced by the injected ions, as a function of position within the expander is shown in Figure 3.

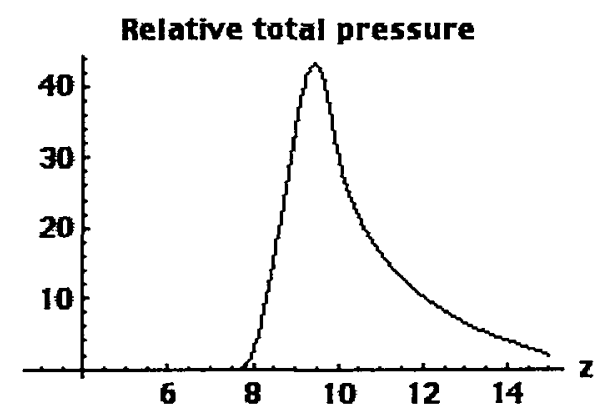

Figure 3: Plot of ion density of peaked plasma (relative to density at the sources)

Evaluating the stability integral for the peaked plasma (for unit pressure at the location of the ion sources) the value +408.0 was found. Comparing this value to the value of $-1.4 \times 10^{-4}$ from Table $I$ that was found for a sloshing ion distribution in the plug cell of a tandem mirror, it can be seen that the ion pressure at the surface where the ion sources are located can be more than six orders of magnitude smaller than that in the plugs and still be sufficient to $\mathrm{MHD}$-stabilize them. As a consequence the ion beam power required to stabilize a tandem-mirror fusion power plant based on the original Dimov/FowlerLogan concept (potentials produced solely by high-density plasmas in the plug cells) would still be small compared to the fusion power output..

\section{8) ADDITIONAL OPTIONS}

In addition to the mode of operation that involves the injection of ion beams up the magnetic gradient, another optional mode of operation has been studied. This mode of operation could be advantageously employed after the plasma of a tandem-mirror system has been stabilized by the K-S beams and the plasma potentials has been formed. At this point in time, as illustrated schematically in Figure 4 , directed gas jets would be turned on, aimed tangentially at the periphery of the plasma at a point near the mirror. The jets would be located on the down-hill side of the ambipolar potential peak in the plug cell, for example at a point where the potential with respect to that at the end of the expander is about $1.0 \mathrm{kV}$. The plasma at the point of injection of the jets would then ionize their atoms, resulting in an accelerated stream of $1.0 \mathrm{keV}$ ions flowing out into the expander. This stream would then take over the stabilization, allowing the stabilizer ion sources to be turned off, thus simplifying the stabilization process. 


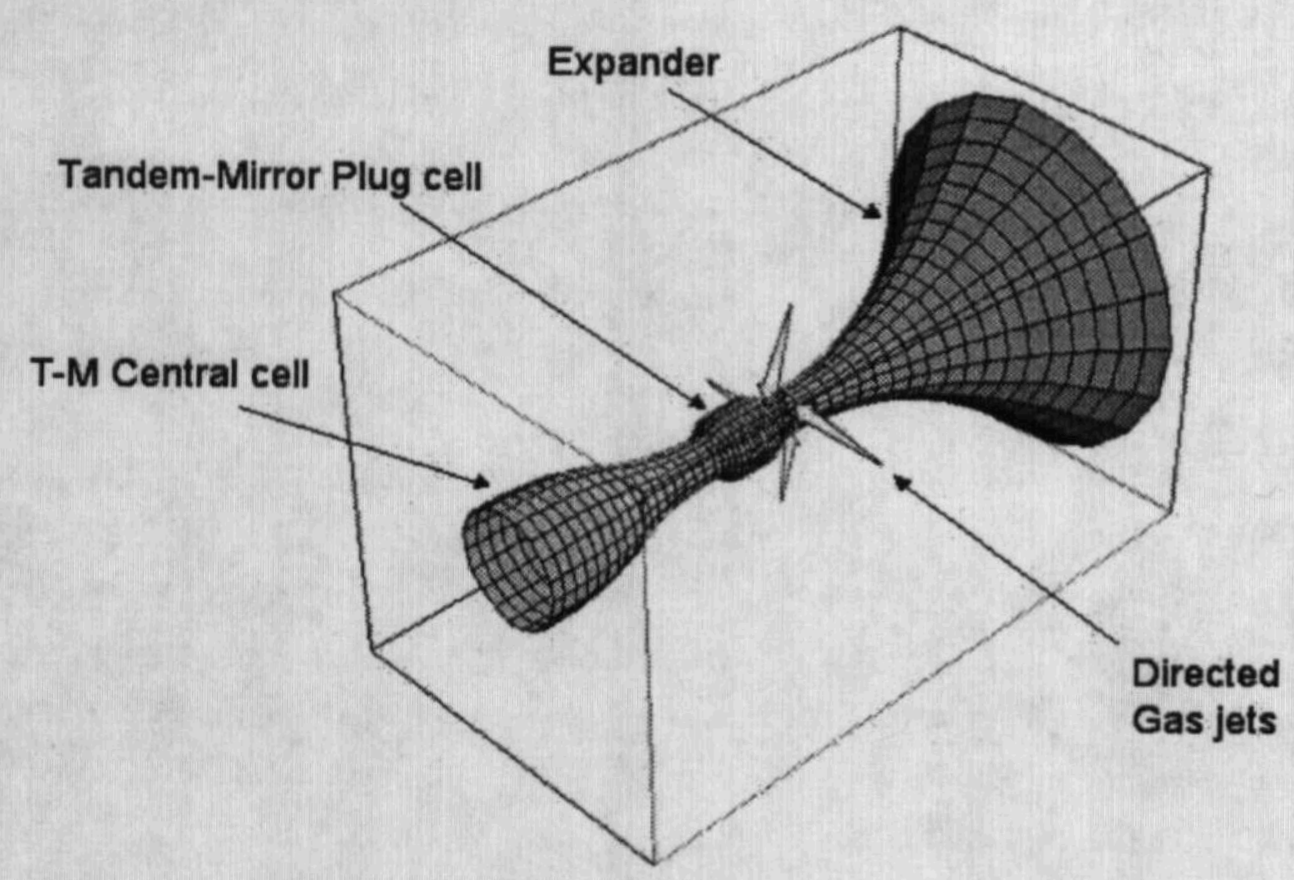

Figure 4: Schematic drawing of gas jet stabilizer beams and tandem-mirror flux surfaces.

The stability integral code, modified to allow the introduction of an ion stream (of krypton ions) originating near the mirror, was run to find the value of the instability integral. In a typical case the value found for $\mathrm{I}_{\mathrm{s}}$, (for unit pressure of the ion stream at its origin) was +0.28 . This value is about four orders of magnitude greater than the value of $I_{s}$ for the plug, implying that the kinetic pressure of the ion stream could be four orders of magnitude smaller than that of the plug plasma and still stabilize. When the area of the flux tube near the mirror throat (where the streams are formed) was factored in, in a typical tandemmirror example it was found that the power extracted by the stabilizer streams was only about $200 \mathrm{~kW}$, thus was far less than the power required to maintain the plug, which itself was much less than the fusion power release.

\section{9) WORK IN PROGRESS}

As of the writing of this report there are several other activities in progress in support of analyzing the Kinetic Stabilizer concept. Two legacy computer codes, FLORA and MCPAT, written during the 1980 s as a part of the tandem-mirror program at the Laboratory, are being updated to enable them to be run on modern work stations. FLORA is an initial-value MHD stability code which includes finite-beta capabilities. MCPAT is a MonteCarlo-based code that can be used to investigate electron physics and collisional effects in the Kinetic Stabilizer Tandem Mirror system. Also being investigated are the plasma communication issues. These issues refer to the necessity of insuring sufficient conductivity in the region between the plug cell and the stabilizer plasma peak to avoid the so-called trapped-ion or other communicationrelated effects.

In addition to the code updating, some studies are being made of the application of LLNL-developed liquid- wall concepts [20] to the KS/TM. Because of its axisymmetry the KS/TM seems well suited to introducing liquid walls whose purpose is to absorb the power generated in the fusing plasma and transport this resulting heat to the conventional part of the power plant where the heat is converted into electricity. The liquid is kept from falling into the plasma by centrifugal force of azimuthal motion. If the liquid is the molten salt, flibe (Li2BeF4) about $0.5 \mathrm{~m}$ thick ( 7 mean free paths for $14 \mathrm{MeV}$ neutrons), the structures are predicted to last the life of the power plant, being limited by neutron radiation damage. The use of liquid walls solves the "first wall" problem. The economic benefits of successful liquid walls to a fusion plant are multiple: higher power density, less down time due to not changing out structures, less building space from avoiding these change-outs and less radioactive structures to handle. The open-ended nature of the KS/TM facilitates introducing and extracting the flowing liquid. The edge plasma will (and must) sufficiently protect the core plasma from too much contamination by the evaporating liquid. The feasibility of using liquid walls will rest on the contamination being kept to under about $1 \%$ fluorine contaminant in the core plasma. If analysis and experiments prove contamination is acceptably low, liquid walls could significantly improve the power plant competitiveness of the $\mathrm{KS} / \mathrm{TM}$ relative to other power plants.

\section{0) SUMMARY AND CONCLUSIONS}

The history of magnetic fusion research has shown us that open systems with axisymmetric fields have the potential to confine plasma in near-quiescent states, with cross-field transport rates approaching classical values. Undergirded by theory, experiments performed on the Gas Dynamic Trap at Novosibirsk show the way to stabilizing axisymmetric mirror systems against MHD interchange modes. The Kinetic Stabilizer concept and its variations represent a way to implement the same stabilization concept in a tandem-mirror system based on the original Dimov/Fowler-Logan concept. Following this path may lead to simpler, smaller magnetic fusion power system the development of which might be much faster and less costly than the path represented by the tokamak or other closed-field approaches.

\section{1) ACKNOWLEDGEMENTS}

This work was performed under the auspices of the U. S. Department of Energy by the University of California Lawrence Livermore National Laboratory under contract W-7405-ENG-48. The author would like to acknowledge with thanks the help of Dmitri Ryutov in plasma physics issues connected with the K-S concept, the work of Bruce Cohen and Jack Byers in updating the FLORA code and Tom Rognlien with the MCPAT code, the discussions with 
Herbert Berk (University of Texas) concerning trapped-ion modes and related issues, and the comments by Ralph Moir on his work on liquid blankets as they might be applied in the KS/TM.

\section{2) REFERENCES}

[1] R. F. Post, Trans. of Fusion Technology 39, 25 (2001)

[2] H. Alfv n, Cosmical Electrodynamics, Oxford Press (1948)

[3] L Spitzer, Physics of Fully Ionized Gases, Interscience (1962)

[4] M. N. Rosenbluth, C. L. Longmire, Ann. Physics 1, 120 (1957)

[5] M. S. Ioffe, R. I Sobolev, et. al., Sov. Phys. - JETP 12,1117 (1961)

[6] M. N Rosenbluth, R. F. Post, Phys Fluids 8, 547 (1965)

[7] D. L. Correll, J. F. Clauser, F. H. Coensgen, et. al., Nuclear Fusion 20, 655 (1980)

[8] R. W. Moir, W. L. Barr, G. A. Carlson, in Plasma Physics and Controlled Nuclear Fusion Research 1974, Vol.3, IAEA, Vienna, 583 (1975)

[9] L. A. Artsimovich, et. al., Plasma Physics and Controlled Nuclear Fusion Research 1968, IAEA, Vienna, 157 (1969)
[10] G. I. Dimov, V. V. Zakaidakov, M. E. Kishinevskii, Sov. J. Plasma Phys. 2, 326 (1976)

[11] T. K. Fowler, B. G. Logan, Comments Plasma Phys. Controll. Fusion 2,167 (1977)

[12] D. D. Ryutov, Axisymmetric MHD-Stable Mirrors, Proceedings of the Course and Workshop, Varenna, Italy, Vol. II,791 (1987)

[13] P. A. Bagryansky, et. al., Trans. of Fusion Technology 35, 79 (1999)

[14] R. F. Post, R. E. Ellis, F. C. ford, M. N. Rosenbluth, Phys. Rev. Lett. 4, 166 (1964)

[15] R. F. Post, Trans. of Fusion Technology 35, 40 (1999)

[16] R. F. Post, Nuclear Fusion 27, 1579 (1987)

[17] T. I. Orzechowski, S. L. Allen, et. al., Phys. Fluids 26, 2335 (1983)

[18] R. F. Post in Plasma Physics Reports (in press) [19] V.V. Mirnov, D.D. Ryutov, Gas Dynamic Trap, pp.77-130, in: Plasma Physics, Vol.8 (Summaries in Science and Technology, V.D. Shrafanov, Ed.), State Institute for Scientific Information, Moscow, 1988 (in Russian)

[20] R. W. Moir (private communication) 